\title{
$\mathrm{Fe}_{3} \mathrm{O}_{4}$ THICK FILMS AS DESCRIBED BY SECOND ORDER PERTURBED HEISENBERG HAMILTONIAN
}

\section{P. SAMARASEKARA}

\section{Department of Physics, University of Peradeniya, Peradeniya, Sri Lanka}

Received 19th November 2019 / Accepted 20th February 2020

\begin{abstract}
Modified second order perturbed Heisenberg Hamiltonian has been used to explain magnetic properties including magnetic easy axis orientation of $\mathrm{Fe}_{3} \mathrm{O}_{4}$ thick films up to 1000 spin layers. The variation of magnetic easy and hard directions with the number of spin layers and stress induced anisotropy was investigated. 3-D plot of energy versus angle and number of spin layers was plotted to find the values of number of spin layers corresponding to energy minima and maxima. By plotting the curve of energy versus angle at one selected value of number of layers, magnetic easy and hard directions were determined. Similarly 3-D plot of energy versus angle and stress induced anisotropy was plotted to find the values of stress induced anisotropy corresponding to energy minima and maxima. Then the curve of energy versus angle at one selected value of stress induced anisotropy was plotted to find the magnetic easy and hard directions. MATLAB software was employed to plot all the graphs.
\end{abstract}

Keywords: spin, stress induced anisotropy, second order perturbed Heisenberg Hamiltonian, ferrites, magnetic easy direction

\footnotetext{
*Corresponding author Email: pubudus@pdn.ac.lk;

D http://orcid.org/0000-0003-2806-0518

DOI: http:://doi.org/10.4038/josuk.v13i0.8010

This is an open access article distributed under the terms of the Creative Commons Attribution 4.0 International License, which permits unrestricted use, distribution and reproduction in any medium provided the original work are properly credited.
} 


\section{P. Samarasekara}

\section{INTRODUCTION}

$\mathrm{Fe}_{3} \mathrm{O}_{4}$ (magnetite) is a prime candidate in applications of magnetic storage, industrial catalysts, water purification and drug delivery. $\mathrm{Fe}_{3} \mathrm{O}_{4}$ is a non-uniaxial ferrite with inverse spinel structure. Magnetic easy axis of $\mathrm{Fe}_{3} \mathrm{O}_{4}$ is along one of the body diagonal of the cubic cell due to the spin arrangement parallel and antiparallel to the body diagonal. Spinel structure with tetrahedral and octahedral sites can be found in detail in some previous publications (Ahmed Farag et al., 2001a, Ahmed Farag et al., 2001b, Kahlenberg et al., 2001, John Zhang et al., 1998, Sickafus et al., 1999). Five of $\mathrm{Fe}^{3+}$ ions occupy tetrahedral sites. Other five $\mathrm{Fe}^{3+}$ ions and four $\mathrm{Fe}^{2+}$ ions occupy octahedral sites. The spinel structure of this ferrite is represented by $\mathrm{Fe}^{3+(} \mathrm{Fe}^{2+} \mathrm{Fe}^{3+)} \mathrm{O}_{4}$. The magnetic moments of $\mathrm{Fe}^{2+}$ and $\mathrm{Fe}^{3+}$ are $4 \mu_{\mathrm{B}}$ and $5 \mu_{\mathrm{B}}$, respectively. Because magnetic moments of $\mathrm{Fe}^{3+}$ in tetrahedral and octahedral sites cancel each other, the net magnetic moment of $\mathrm{Fe}_{3} \mathrm{O}_{4}$ is completely due to the magnetic moments of four $\mathrm{Fe}^{2+}$ ions. Therefore, the theoretical net magnetic moment of $\mathrm{Fe}_{3} \mathrm{O}_{4}$ is $4 \mathrm{Bohr}$ magnetons.

Rietveld method has been employed to determine the cation distribution of ferrite like compounds (Ahmed Farag et al., 2001a, Ahmed Farag et al., 2001b). Surface spin waves in $\mathrm{CsCl}$ type ferrimagnet with a (001) surface has been studied by combining Green function theory with the transfer matrix method (Dai \& Li, 1990). Anisotropy of ultrathin ferromagnetic films and the spin reorientation transition have been investigated using Heisenberg Hamiltonian with few terms (Usadel \& Hucht, 2002). In addition, the surface magnetism of ferrimagnet thin films has been studied using Heisenberg method (Ding et al., 1993). The surface spin wave spectra of both the simple cubic and body centered ferrimagnets have been theoretically studied using Heisenberg Hamiltonian (Hung et al., 1975). The cation distribution and oxidation state of Mn-Fe spinel nanoparticles have been systematically studied at various temperatures by using neutron diffraction and electron 
energy loss spectroscopy (John Zang et al., 1998). The crystal structure of spinel type compounds has been found using single crystal X-ray diffraction data (Kahlenberg et al., 2001). Surface spin waves on the (001) free surface of semi-infinite two lattice ferrimagnets on the Heisenberg model with nearest neighbor exchange interactions has been investigated (Zheng \& Lin, 1988). The lattice parameter, anion parameter and the cation inversion parameter of spinel structures have been presented (Sickafus et al., 1999).

Ferromagnetic ultra thin and thick films have been studied previously using third order perturbed Heisenberg Hamiltonian (Samarasekara, 2008, Samarasekara \& Mendoza, 2010). Ferromagnetic ultra-thin and thick films have been investigated using second order perturbed Heisenberg Hamiltonian by us (Samarasekara \& Gunawardhane, 2011). Furthermore, ferrite ultra-thin and thick films have been investigated using second order perturbed Heisenberg Hamiltonian by us (Samarasekara et al., 2009, Samarasekara, 2010). Ferrite ultra-thin and thick films have been investigated using third order perturbed Heisenberg Hamiltonian (Samarasekara \& Mendoza, 2011, Samarasekara, 2011). The magnetic properties of the spinel $\mathrm{Fe}_{3} \mathrm{O}_{4}$ thick films are described in this manuscript.

\section{MODEL}

The modified Heisenberg Hamiltonian of any thin film with $N$ spin layers can be given as

$$
\begin{aligned}
\mathrm{H}= & -J \sum_{m, n} \vec{S}_{m} \cdot \vec{S}_{n}+\omega \sum_{m \neq n}\left(\frac{\vec{S}_{m} \cdot \vec{S}_{n}}{r_{m n}{ }^{3}}-\frac{3\left(\vec{S}_{m} \cdot \vec{r}_{m n}\right)\left(\vec{r}_{m n} \cdot \vec{S}_{n}\right)}{r_{m n}{ }^{5}}\right)-\sum_{m} D_{\lambda_{m}}{ }^{(2)}\left(S_{m}{ }^{z}\right)^{2}-\sum_{m} D_{\lambda_{m}}{ }^{(4)}\left(S_{m}{ }^{z}\right)^{4} \\
& -\sum_{m} \vec{H} \cdot . \vec{S}_{m}-\sum_{m} K_{s} \operatorname{Sin} 2 \theta_{m}
\end{aligned}
$$

Here $J$ is spin exchange interaction, $\omega$ is the strength of long range dipole interaction, $\theta$ is azimuthal angle of spin, $D_{m}{ }^{(2)}$ and $D_{m}{ }^{(4)}$ are second and fourth order 


\section{P. Samarasekara}

anisotropy constants, $H_{\text {in }}$ and $H_{\text {out }}$ are in plane and out of plane applied magnetic fields, $K_{s}$ is stress induced anisotropy constant, $n$ and $m$ are spin plane indices and $N$ is total number of layers in film. When the stress applies normal to the film plane, the angle between $m^{\text {th }}$ spin and the stress is $\theta_{m}$.

The spinel cubic cell can be divided into 8 spin layers with alternative $\mathrm{Fe}^{2+}$ and $\mathrm{Fe}^{3+}$ spins layers (Sickafus et al., 1999). The spins in one layer and adjacent layers point in one direction and opposite directions, respectively. The spins of $\mathrm{Fe}^{2+}$ and $\mathrm{Fe}^{3+}$ will be taken as 1 and $p$, respectively. A cubic unit cell with length $a$ will be considered. Due to the super exchange interaction between spins, the spins are parallel or antiparallel to each other within the cell. Therefore the results proven for oriented case in one of our early report (Samarasekara et al., 2009) will be used for following equations. But the angle $\theta$ will vary from $\theta_{m}$ to $\theta_{m+1}$ at the interface between two cells.

Following equations will be proven for a thin film with thickness $\mathrm{Na}$.

Spin exchange interaction energy $=E_{\text {exchange }}=N\left(-10 J+72 J p-22 J p^{2}\right)+8 J p \sum_{m=1}^{N-1} \cos \left(\theta_{m+1}-\theta_{m}\right)$

Dipole interaction energy $=E_{\text {dipole }}$

$$
E_{\text {dipole }}=-48.415 \omega \sum_{m=1}^{N}\left(1+3 \cos 2 \theta_{m}\right)+20.41 \omega p \sum_{m=1}^{N-1}\left[\cos \left(\theta_{m+1}-\theta_{m}\right)+3 \cos \left(\theta_{m+1}+\theta_{m}\right)\right]
$$

The first and second term in each above equation represent the variation of energy within the cell (Samarasekara et al., 2009) and the interface of the cell, respectively.

Total energy 


$$
\begin{aligned}
E= & N\left(-10 J+72 J p-22 J p^{2}\right)+8 J p \sum_{m=1}^{N-1} \cos \left(\theta_{m+1}-\theta_{m}\right) \\
& -48.415 \omega \sum_{m=1}^{N}\left(1+3 \cos 2 \theta_{m}\right)+20.41 \omega p \sum_{m=1}^{N-1}\left[\cos \left(\theta_{m+1}-\theta_{m}\right)+3 \cos \left(\theta_{m+1}+\theta_{m}\right)\right] \\
& -\sum_{m=1}^{N}\left[D_{m}{ }^{(2)} \cos ^{2} \theta_{m}+D_{m}{ }^{(4)} \cos ^{4} \theta_{m}\right] \\
& -4(1-p) \sum_{m=1}^{N}\left[H_{\text {in }} \sin \theta_{m}+H_{\text {out }} \cos \theta_{m}+K_{s} \sin 2 \theta_{m}\right]
\end{aligned}
$$

The anisotropy energy term and the last term given in above equations have been explained in our previous report for oriented spinel ferrite (Samarasekara et al., 2009). The angle can be given as $\theta_{m}=\theta+\varepsilon_{m}$ with perturbation $\varepsilon_{m}$.

The total energy can be given as $E(\theta)=E_{0}+E(\varepsilon)+E\left(\varepsilon^{2}\right)$

Here the energy is given only up to the second order perturbation of $\varepsilon$. Then after expanding sin and cosine terms,

$$
\begin{aligned}
& E_{0}=-10 J N+72 p N J-22 J p^{2} N+8 J p(N-1)-48.415 \omega N-145.245 \omega N \cos (2 \theta) \\
& +20.41 \omega p[(N-1)+3(N-1) \cos (2 \theta)] \\
& \quad-\cos ^{2} \theta \sum_{m=1}^{N} D_{m}{ }^{(2)}-\cos ^{4} \theta \sum_{m=1}^{N} D_{m}{ }^{(4)}-4(1-p) N\left(H_{\text {in }} \sin \theta+H_{\text {out }} \cos \theta+K_{s} \sin 2 \theta\right) \\
& E(\varepsilon)=290.5 \omega \sin (2 \theta) \sum_{m=1}^{N} \varepsilon_{m}-61.23 \omega p \sin (2 \theta) \sum_{m=1}^{N-1}\left(\varepsilon_{m}+\varepsilon_{n}\right) \\
& \quad+\sin 2 \theta \sum_{m=1}^{N} D_{m}{ }^{(2)} \varepsilon_{m}+2 \cos ^{2} \theta \sin 2 \theta \sum_{m=1}^{N} D_{m}{ }^{(4)} \varepsilon_{m}
\end{aligned}
$$




$$
\begin{gathered}
+4(1-p)\left[-H_{\text {in }} \cos \theta \sum_{m=1}^{N} \varepsilon_{m}+H_{\text {out }} \sin \theta \sum_{m=1}^{N} \varepsilon_{m}-2 K_{s} \cos 2 \theta \sum_{m=1}^{N} \varepsilon_{m}\right] \\
E\left(\varepsilon^{2}\right)=-4 J p \sum_{m=1}^{N-1}\left(\varepsilon_{n}-\varepsilon_{m}\right)^{2}+290.5 \omega \cos (2 \theta) \sum_{m=1}^{N} \varepsilon_{m}{ }^{2}-10.2 \omega p \sum_{m=1}^{N-1}\left(\varepsilon_{n}-\varepsilon_{m}\right)^{2} \\
-30.6 \omega p \cos (2 \theta) \sum_{m=1}^{N-1}\left(\varepsilon_{n}+\varepsilon_{m}\right)^{2} \\
-\left(\sin ^{2} \theta-\cos { }^{2} \theta\right) \sum_{m=1}^{N} D_{m}{ }^{(2)} \varepsilon_{m}{ }^{2}+2 \cos { }^{2} \theta\left(\cos ^{2} \theta-3 \sin ^{2} \theta\right) \sum_{m=1}^{N} D_{m}{ }^{(4)} \varepsilon_{m}{ }^{2} \\
+4(1-p)\left[\frac{H_{\text {in }}}{2} \sin \theta \sum_{m=1}^{N} \varepsilon_{m}{ }^{2}+\frac{H_{o u t}}{2} \cos \theta \sum_{m=1}^{N} \varepsilon_{m}{ }^{2}+2 K_{s} \sin ^{2} \theta \sum_{m=1}^{N} \varepsilon_{m}{ }^{2}\right]
\end{gathered}
$$

Here $n=m+1$.

After using constraint $\sum_{m=1}^{N} \varepsilon_{m}=0$, first and last three terms of equation 4 are zero.

Then, $\mathrm{E}(\varepsilon)=\vec{\alpha} \cdot \vec{\varepsilon}$

where $\vec{\alpha}(\varepsilon)=\vec{B}(\theta) \sin 2 \theta$ are the terms of matrices with

$B_{\lambda}(\theta)=-122.46 \omega p+D_{\lambda}^{(2)}+2 D_{\lambda}^{(4)} \cos ^{2} \theta$

Also $E\left(\varepsilon^{2}\right)=\frac{1}{2} \vec{\varepsilon} \cdot C \cdot \vec{\varepsilon}$

where the elements of matrix $C$ can be given as following,

$C_{m, m+1}=8 J p+20.4 \omega p-61.2 p \omega \cos (2 \theta)$

For $m=1$ and $N$, 


$$
\begin{aligned}
C_{m m} & =-8 J p-20.4 \omega p-61.2 p \omega \cos (2 \theta)+581 \omega \cos (2 \theta)-2\left(\sin ^{2} \theta-\cos ^{2} \theta\right) D_{m}^{(2)} \\
& +4 \cos ^{2} \theta\left(\cos ^{2} \theta-3 \sin ^{2} \theta\right) D_{m}^{(4)}+4(1-p)\left[H_{\text {in }} \sin \theta+H_{\text {out }} \cos \theta+4 K_{s} \sin (2 \theta)\right]
\end{aligned}
$$

For $m=2,3,----, N-1$

$$
\begin{aligned}
C_{m m} & =-16 J p-40.8 \omega p-122.4 p \omega \cos (2 \theta)+581 \omega \cos (2 \theta)-2\left(\sin ^{2} \theta-\cos ^{2} \theta\right) D_{m}{ }^{(2)} \\
& +4 \cos ^{2} \theta\left(\cos ^{2} \theta-3 \sin ^{2} \theta\right) D_{m}{ }^{(4)}+4(1-p)\left[H_{\text {in }} \sin \theta+H_{\text {out }} \cos \theta+4 K_{s} \sin (2 \theta)\right]
\end{aligned}
$$

Otherwise, $C_{m n}=0$

Finally the total energy can be given as

$$
E(\theta)=E_{0}+\vec{\alpha} \cdot \vec{\varepsilon}+\frac{1}{2} \vec{\varepsilon} \cdot C \cdot \vec{\varepsilon}=E_{0}-\frac{1}{2} \vec{\alpha} \cdot C^{+} \cdot \vec{\alpha}
$$

Here $C^{+}$is the pseudo-inverse given by

$$
C . C^{+}=1-\frac{E}{N} .
$$

For a thick film with $N=10000$, the second term in equation number 9 can be neglected.

Then $C^{+}$will be the normal inverse of matrix $C$.

\section{RESULTS AND DISCUSSION}

If anisotropy constants $D_{m}{ }^{(2)}$ and $D_{m}{ }^{(4)}$ do not vary within the film, then $C_{11}=$ $C_{N N}$ and $C_{22}=C_{33}=------=C_{N-1, N-1}$. When $H_{\text {in }}, H_{\text {out }}$ and $K_{s}$ are very large, $C_{22}>>C_{12}$. If $C_{m, m+1}$ is zero, then the matrix $C$ will be diagonal. Then the elements of inverse matrix $\left(C^{+}\right)$ 


\section{P. Samarasekara}

is given by $C^{+}{ }_{m m}=\frac{1}{C_{m m}}$. To avoid tedious calculations, the solution will be found under assumption $C_{m, m+1}=0$.

Then $\alpha_{1}=---=\alpha_{n}=\left[-122.46 \omega p+D_{\lambda}{ }^{(2)}+2 D_{\lambda}{ }^{(4)} \cos ^{2} \theta\right] \sin (2 \theta)$

$\alpha \cdot C^{+} \cdot \alpha=2 C^{+}{ }_{11} \alpha_{1}^{2}++\alpha_{1}{ }^{2}(N-2) C^{+}{ }_{22}=\frac{2 \alpha_{1}^{2}}{C_{11}}+\frac{\alpha_{1}^{2}(N-2)}{C_{22}}$

For $\mathrm{Fe}_{3} \mathrm{O}_{4}, p=5 / 4=1.25$.

From equation 3,

$E_{0}=55.625 N J-10 J-48.415 \omega N-145.245 \omega N \cos (2 \theta)+25.5125 \omega(N-1)[1+3 \cos (2 \theta)]$

$$
-N\left[\cos ^{2} \theta D_{m}^{(2)}+\cos ^{4} \theta D_{m}^{(4)}-\left(H_{\text {in }} \sin \theta+H_{o u t} \cos \theta+K_{S} \sin 2 \theta\right)\right]
$$

From equation 7 ,

$$
\begin{gathered}
C_{11}=C_{N N}=-10 J-25.5 \omega+504.5 \omega \cos (2 \theta)+2 \cos 2 \theta D_{m}{ }^{(2)}+4 \cos ^{2} \theta\left(\cos ^{2} \theta-3 \sin ^{2} \theta\right) D_{m}{ }^{(4)} \\
\quad-\left[H_{\text {in }} \sin \theta+H_{\text {out }} \cos \theta+4 K_{S} \sin (2 \theta)\right] \\
C_{22}=C_{33}=----C_{N-1, N-1}=-20 J-51 \omega+428 \omega \cos (2 \theta)+2(\cos 2 \theta) D_{m}{ }^{(2)} \\
+4 \cos ^{2} \theta\left(\cos ^{2} \theta-3 \sin ^{2} \theta\right) D_{m}{ }^{(4)}-\left[H_{\text {in }} \sin \theta+H_{\text {out }} \cos \theta+4 K_{S} \sin (2 \theta)\right] \\
\alpha_{1}=\left[-153.08 \omega+D_{\lambda}{ }^{(2)}+2 D_{\lambda}{ }^{(4)} \cos ^{2} \theta\right] \sin (2 \theta)
\end{gathered}
$$

From equation 8 ,

Total energy $=E(\theta)=E_{0^{-}} \frac{\alpha_{1}^{2}}{C_{11}}-\frac{\alpha_{1}^{2}(N-2)}{2 C_{22}}$ 
Figure 1 shows the 3-D plot of $\frac{E(\theta)}{\omega}$ versus $\theta$ and $\mathrm{N}$, for $\frac{J}{\omega}=\frac{D_{m}{ }^{(2)}}{\omega}=\frac{H_{\text {in }}}{\omega}=\frac{H_{\text {out }}}{\omega}=\frac{K_{s}}{\omega}=10$ and $\frac{D_{m}^{(4)}}{\omega}=5 . \quad$ Several energy minima and maxima can be observed at different values of N. According to this graph, the magnetic easy direction depends on the number of spin layers or the thickness of the film. In addition, the variation of magnetic easy direction with the deposition temperature has been explained using Heisenberg Hamiltonian coupled with spin reorientation by us (Samarasekara \& Gunawardhane, 2011, Samarasekara \& Saparamadu, 2012, Samarasekara \& Saparamadu, 2013). This graph is similar to the same graph plotted for thick ferromagnetic films using third order perturbed Heisenberg Hamiltonian, and the maximum value of energy is also the same in the both cases (Samarasekara, 2008). The same graph plotted for thick nickel ferrite films using second order perturbed Heisenberg Hamiltonian is similar to this graph (Samarasekara, 2010). 


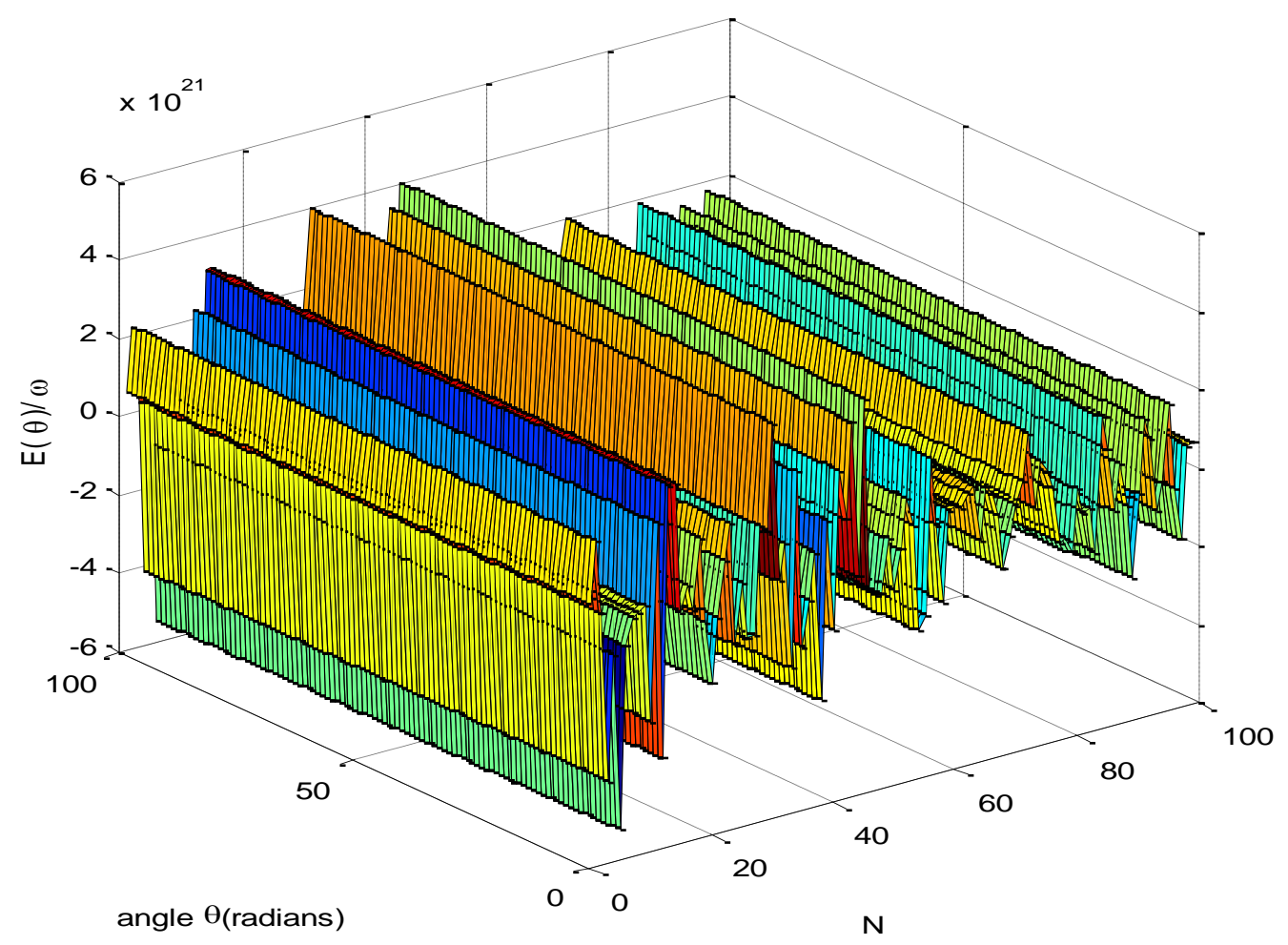

Figure 1: 3-D plot of $\frac{E(\theta)}{\omega}$ versus $\theta$ and $\mathrm{N}$

The graph of $\frac{E(\theta)}{\omega}$ versus $\mathrm{N}$ is given in figure 2 for $\theta=0$ radians. Major energy minima can be observed at $\mathrm{N}=5,12,20$, ------ etc. Major energy maxima can be observed at $\mathrm{N}=1$ $5,19,24$, ---- etc. This graph can be obtained by rotating above 3-D plot in MATLAB. In addition to major minima and maxima, some minor maxima and minima can be observed in figure 2. 


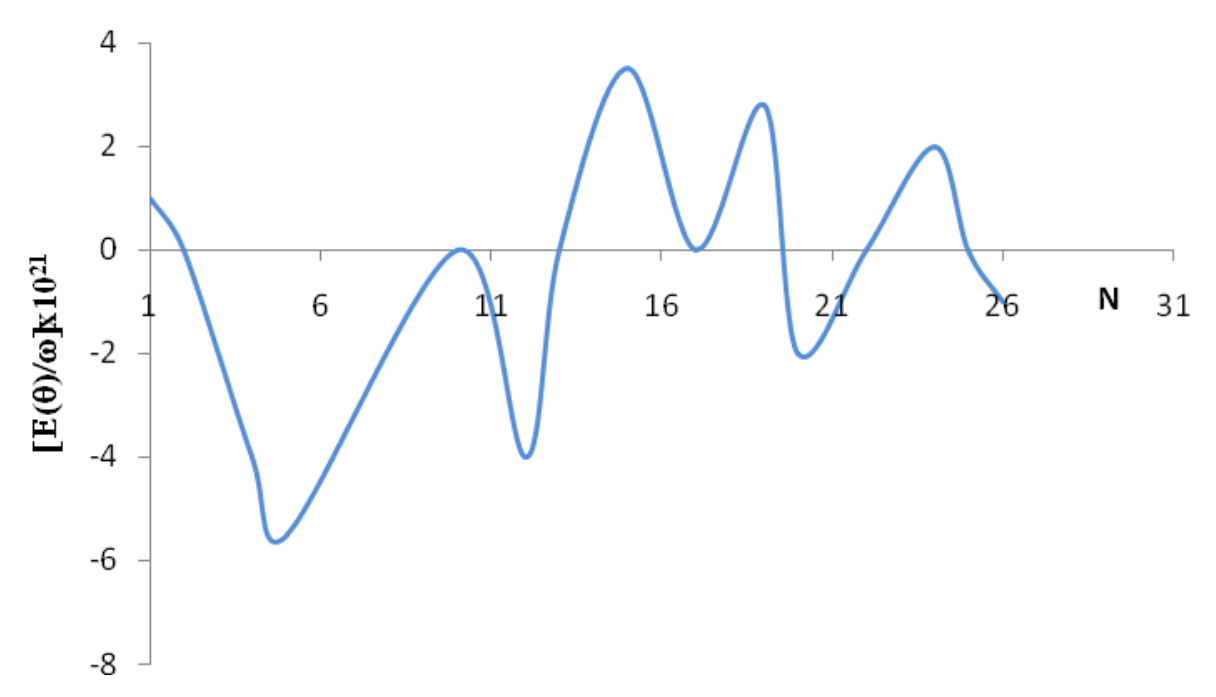

Figure 2: Graph of $\frac{E(\theta)}{\omega}$ versus $\mathrm{N}$ for $\theta=0$ radians

Figure 3 shows the $\frac{E(\theta)}{\omega}$ versus $\theta$ for $\mathrm{N}=5$. Energy minima of this graph can be observed at $\theta=2.6,5.6$ radians, ----- etc. Energy maxima of this graph can be observed at $\theta=3.6,5.7$ radians,----- etc. Therefore, magnetic easy directions are $\theta=2.6,5.6$ radians, ---- etc with respect to a normal line drawn to the film plane. The same study was done for ultra thin nickel ferrite films with 2 and 3 spin layers using second order perturbed Heisenberg Hamiltonian by the authors (Samarasekara et al., 2009). Broad peaks could be observed for films with 2 spin layers (Samarasekara et al., 2009). However, the same graph plotted for ferromagnetic ultra thin films with three spin layers using third order perturbed Heisenberg Hamiltonian is somewhat similar to the plot given in this case (Samarasekara \& Mendoza, 2010). 


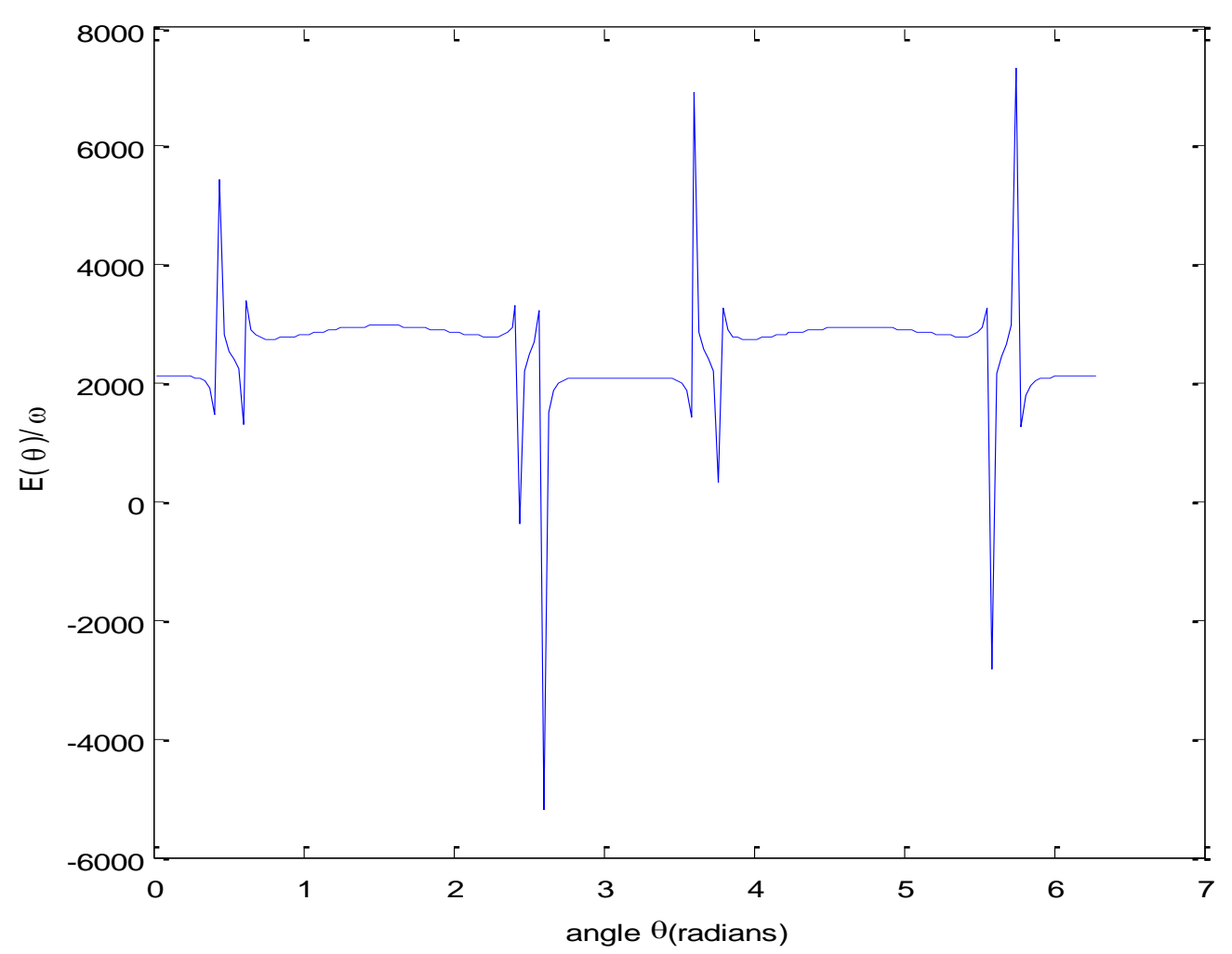

Figure 3: Graph of $\frac{E(\theta)}{\omega}$ versus $\theta$ for $\mathrm{N}=5$

Figure 4 shows the $3-\mathrm{D}$ plot of $\frac{E(\theta)}{\omega}$ versus $\theta$ and $\frac{K_{s}}{\omega}$ for $\mathrm{N}=10000$. The other parameters were kept at $\frac{J}{\omega}=\frac{D_{m}{ }^{(2)}}{\omega}=\frac{H_{\text {in }}}{\omega}=\frac{H_{\text {out }}}{\omega}=10$ and $\frac{D_{m}{ }^{(4)}}{\omega}=5$. Energy minima can be observed at $\frac{K_{s}}{\omega}=17,21,25$------ etc. Energy maxima can be observed at $\frac{K_{s}}{\omega}=19$, 23, 27, ---- etc. The same kind of 3-D graph plotted for ultra thin nickel ferrite films using third order perturbed Heisenberg Hamiltonian is entirely different from the 3-D plot given in this manuscript (Samarasekara \& Mendoza, 2011). However, this graph is similar to the same kind of 3-D graph plotted for nickel ferrite thick films using third order perturbed 
Heisenberg Hamiltonian (Samarasekara, 2011). Figure 5 shows the graph of $\frac{E(\theta)}{\omega}$ versus $\theta$ for $\frac{K_{s}}{\omega}=17$. Energy minima of this graph can be observed at $\theta=2.6$ radians. Energy maxima of this graph can be observed at $\theta=3.6$ radians. Therefore, magnetic easy direction is $\theta=2.6$ radians with respect to a normal line drawn to the film plane. According to our experimental studies, magnetic properties of ferrite films depend on the stress induced anisotropy (Samarasekara \& Cadieu, 2001).

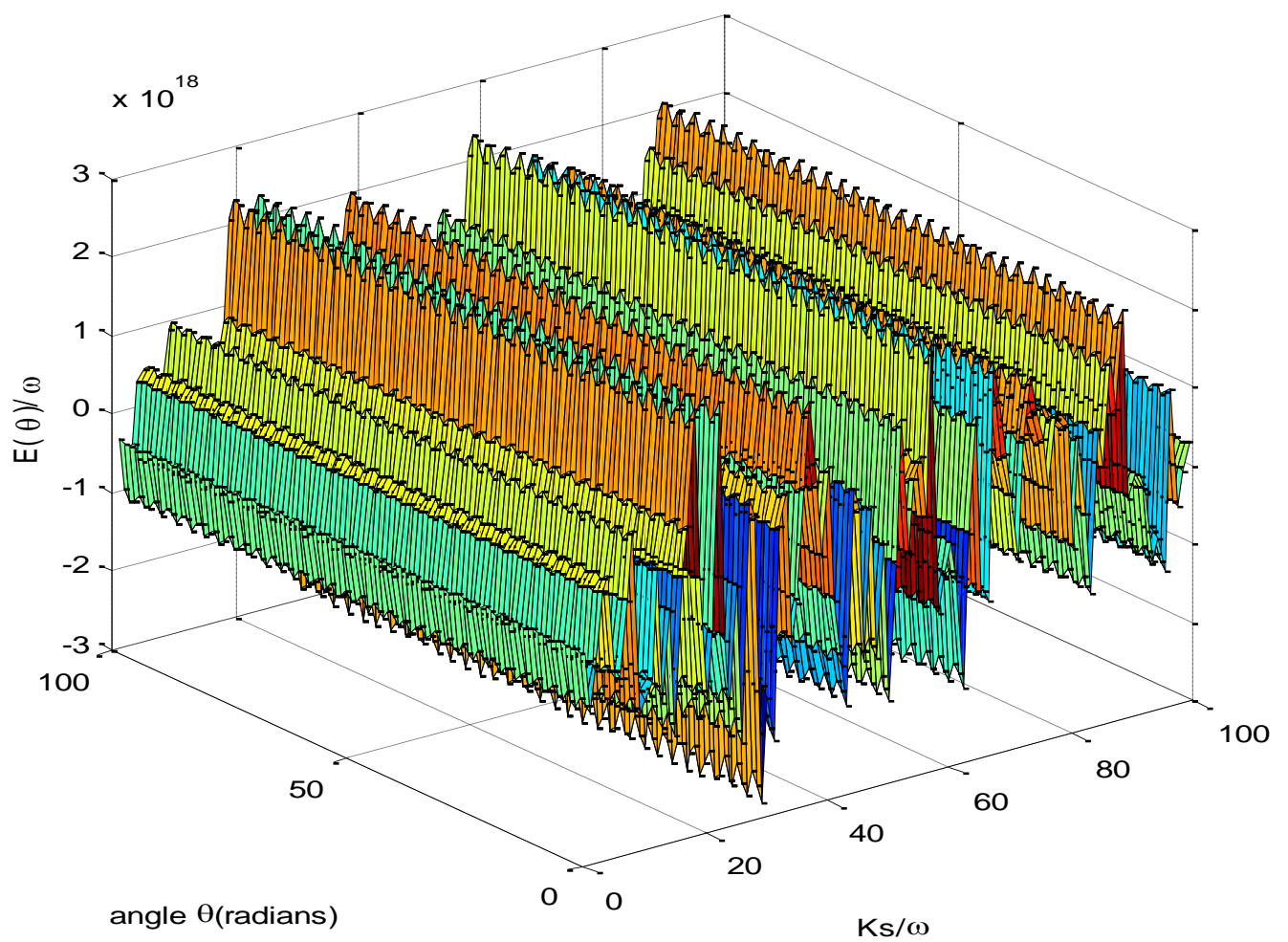

Figure 4: 3-D plot of $\frac{E(\theta)}{\omega}$ versus $\theta$ and $\frac{K_{s}}{\omega}$ for $\mathrm{N}=10000$ 


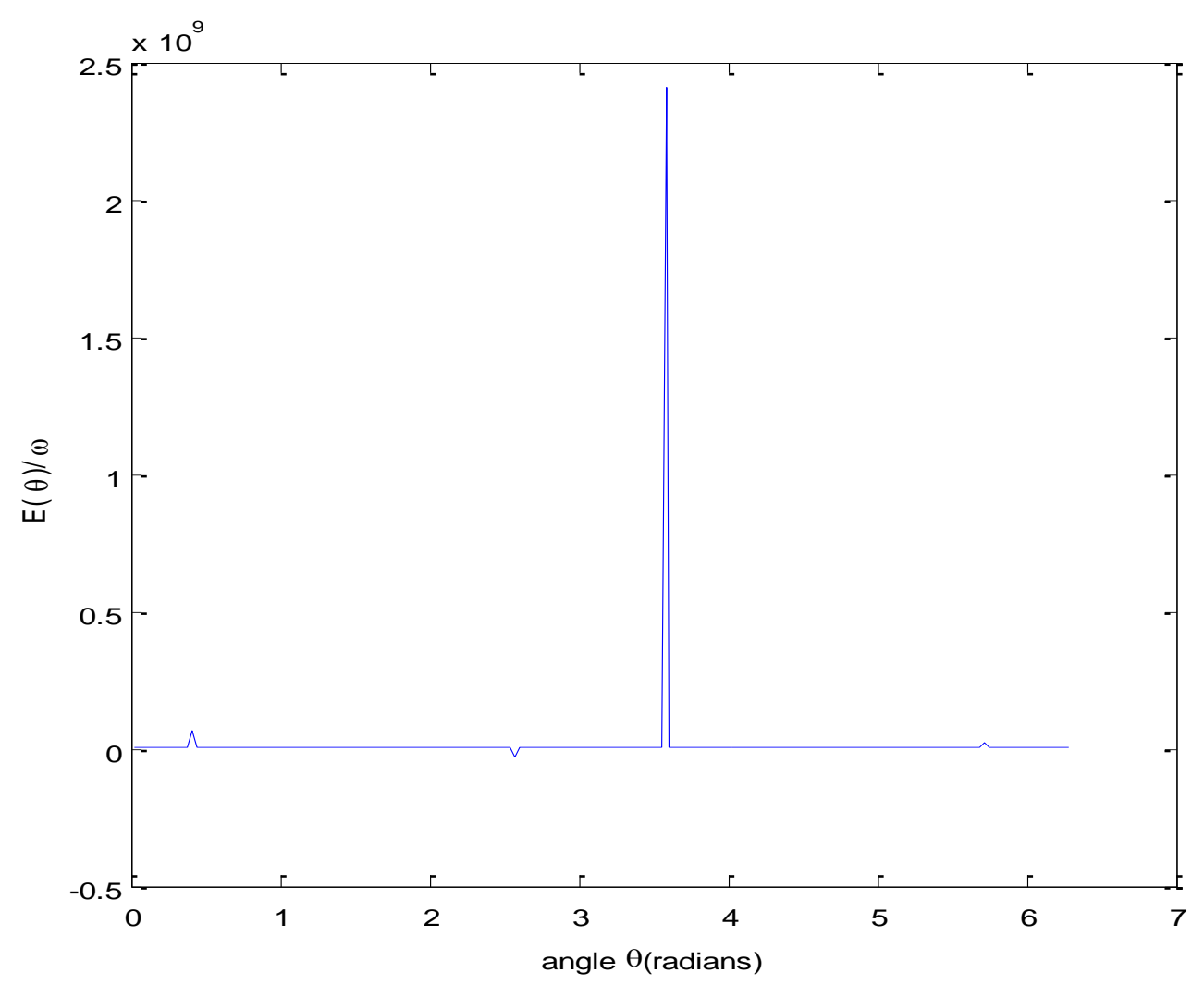

Figure 5: Graph of $\frac{E(\theta)}{\omega}$ versus $\theta$ for $\frac{K_{s}}{\omega}=17$

\section{CONCLUSIONS}

When $\frac{J}{\omega}=\frac{D_{m}{ }^{(2)}}{\omega}=\frac{H_{\text {in }}}{\omega}=\frac{H_{\text {out }}}{\omega}=\frac{K_{s}}{\omega}=10, \frac{D_{m}{ }^{(4)}}{\omega}=5$ and $\mathrm{N}=5$, magnetic easy directions were found to be $\theta=2.6$ radians, 5.6 radians, etc. The angle between two consecutive easy directions is 3 radians (172 degrees). When $\frac{J}{\omega}=\frac{D_{m}{ }^{(2)}}{\omega}=\frac{H_{\text {in }}}{\omega}=\frac{H_{\text {out }}}{\omega}=10, \mathrm{~N}=10000$ and $\frac{K_{s}}{\omega}=17$, magnetic easy direction was $\theta=2.6$ radians. Several magnetic easy and hard directions could be found in first three graphs. According to the 3-D plot of $\frac{E(\theta)}{\omega}$ versus $\theta$ and N, the magnetic easy and hard directions vary with the number of spin layers. Compared to this energy versus angle 
curve for $\mathrm{N}=5$, broad peaks could be observed for films with 2 spin layers. The 3-D graph of $\frac{E(\theta)}{\omega}$ versus $\theta$ and $\frac{K_{s}}{\omega}$ is similar to the same kind of 3-D graph plotted for spinel nickel ferrite thick films using third order perturbed Heisenberg Hamiltonian.

\section{REFERENCES}

Ahmed Farag I.S., Ahmed M.A., Hammad S.M. and Moustafa A.M., 2001a. Study of cation distribution in $\mathrm{Cu}_{0.7}\left(\mathrm{Zn}_{0.3-\mathrm{x}} \mathrm{Mg}_{\mathrm{x}}\right) \mathrm{Fe}_{1.7} \mathrm{Al}_{0.3} \mathrm{O}_{4}$ by $\mathrm{X}$-ray diffraction using Rietveld method. Egyptian Journal of Solids 24(2), 215-225.

Ahmed Farag I.S., Ahmed M.A., Hammad S.M. and Moustafa A.M., 2001b. Application of Rietveld method to the structural characteristics of substituted copper ferrite compounds. Crystal Research \& Technology 36(1), 85-92.

Araya-Pochet J., Ballentine C.A. and Erskine J.L., 1988. Thickness and temperature dependent spin anisotropy of ultrathin epitaxial Fe films on $\operatorname{Ag}(100)$. Physical Review B 38(11), 7846-7849

Dai S.T. and Li Z.Y., 1990. Surface spin waves in a semi-infinite ferrimagnet. Physics Letters A146, 450-456.

Ding Ze-Nong, Lin D.L. and Libin Lin, 1993. Surface magnetism in a thin film of Heisenberg Ferrimagntes. Chinese Journal of Physics 31(3), 431-440.

Hung D.H., Harada I. and Nagai O., 1975. Theory of surface spin-waves in a semi-infinite ferrimagnet. Physics Letters A 53(2), 157-158.

John Zhang Z., Zhong L. Wang, Bryan C. Chakoumakos and Jin S. Yin., 1998. Temperature dependence of cation distribution and oxidation state in magnetic Mn- 


\section{P. Samarasekara}

Fe ferrite nanocrystals. Journal of the American Chemical Society 120(8), 18001804.

Kahlenberg V., Shaw C.S.J. and Parise J.B., 2001. Crystal structure analysis of synthetic $\mathrm{Ca}_{4} \mathrm{Fe}_{1.5} \mathrm{Al}_{17.67} \mathrm{O}_{32}$ : A high-pressure, spinel-related phase. American Mineralogist $86,1477-1482$.

Parlak U., Akoz M.E., Tokdemir Ozturk S. and Erkovan M., 2015. Thickness dependent magnetic properties of polycrystalline nickel thin films. Acta Physica Polonica A 127(4), 995-997.

Samarasekara P. and Cadieu F.J., 2001. Polycrystalline Ni ferrite films deposited by RF sputtering techniques. Japanese Journal of Applied Physics 40, 3176-3179.

Samarasekara P., 2008. Influence of third order perturbation on Heisenberg Hamiltonian of thick ferromagnetic films. Electronic Journal of Theoretical Physics 5(17), 227-236.

Samarasekara P., Abeyratne M.K. and Dehipawalage S., 2009. Heisenberg Hamiltonian with Second Order Perturbation for Spinel Ferrite Thin Films. Electronic Journal of Theoretical Physics 6(20), 345-356.

Samarasekara P., 2010. Determination of Energy of thick spinel ferrite films using Heisenberg Hamiltonian with second order perturbation. Georgian electronic scientific journals: Physics 1(3), 46-52.

Samarasekara P. and William A. Mendoza, 2010. Effect of third order perturbation on Heisenberg Hamiltonian for non-oriented ultra-thin ferromagnetic films. Electronic Journal of Theoretical Physics 7(24), 197-210.

Samarasekara P. and Gunawardhane N.H.P.M., 2011. Explanation of easy axis orientation of ferromagnetic films using Heisenberg Hamiltonian. Georgian electronic scientific journals: Physics 2(6), 62-69. 
Samarasekara P. and William A. Mendoza, 2011. Third Order Perturbed Heisenberg Hamiltonian of Spinel Ferrite Ultra-thin films. Georgian Electronic Scientific Journals:Physics 1(5), 15-24.

Samarasekara P., 2011. Investigation of Third Order Perturbed Heisenberg Hamiltonian of Thick Spinel Ferrite Films. Inventi Rapid: Algorithm Journal 2(1), 1-3.

Samarasekara P. and Saparamadu Udara, 2012. Investigation of Spin Reorientation in Nickel Ferrite Films. Georgian electronic scientific journals: Physics 1(7), 15-20. Samarasekara P. and Saparamadu Udara, 2013. In plane oriented Strontium ferrite thin films described by spin reorientation. Research \& Reviews: Journal of Physics-STM journals 2(2), 12-16.

Sickafus Kurt E., John M. Wills and Norman W. Grimes., 1999. Structure of spinel. Journal of the American Ceramic Society 82(12), 3279-3292.

Usadel K.D. and Hucht A., 2002. Anisotropy of ultrathin ferromagnetic films and the spin reorientation transition. Physical Review B 66, 024419.

Zheng H. and Lin D.L., 1988. Surface spin waves of semi-infinite two sublattice ferrimagnets. Physical Review B 37(16), 9615-9624. 